\title{
On lattice isomorphisms of inverse semigroups, II
}

\author{
Peter R. Jones
}

September 2, 2005

\begin{abstract}
A lattice isomorphism between inverse semigroups $S$ and $T$ is an isomorphism between their lattices of inverse subsemigroups. When $S$ is aperiodic, it has long been known that a bijection is induced between $S$ and $T$. Various authors have introduced successively weaker 'archimedean' hypotheses under which this bijection is necessarily an isomorphism, naturally inducing the original lattice isomorphism. Since lattice-isomorphic groups need not have the same cardinality, extending these techniques to the non-aperiodic case requires some means of tying the subgroups to the rest of the semigroup. Ershova showed that if $S$ has no nontrivial isolated subgroups (subgroups that form an entire $\mathcal{D}$-class) then again a bijection exists between $S$ and $T$. Recently, this technique has been successfully exploited, by Goberstein for fundamental inverse semigroups and by the author for completely semisimple inverse semigroups, under two different 'archimedean' hypotheses. In this paper, we derive further properties of Ershova's bijection(s) and formulate a 'quasi-connected' hypothesis that enables us to derive both Goberstein's and the author's earlier results as corollaries.
\end{abstract}

2000 Mathematics Subject Classification: Primary 20M18; Secondary 08A30

This paper is a sequel to the author's paper [11] and, at the same time, an extension of a recent paper by Goberstein [5], on the extent to which an inverse semigroup $S$ is determined by its lattice $\mathcal{L}(S)$ of inverse subsemigroups: given an $\mathcal{L}$-isomorphism, that is, an isomorphism $\Phi: \mathcal{L}(S) \rightarrow \mathcal{L}(T)$ for some inverse semigroup $T$, how are $S$ and $T$ related? For surveys on this topic, see [10] and [14]. It is easily seen that since $\Phi$ restricts to an $\mathcal{L}$-isomorphism between their respective semilattices of idempotents, $E_{S}$ and $E_{T}$, it induces a bijection $\phi_{E}$ between them. Continuing the context of the cited papers, we focus on the situation where $\phi_{E}$ is an isomorphism, which occurs in a surprising number of contexts, for example (see [8]) when $S$ is simple, when $S$ is $E$-unitary with no trivial $\mathcal{J}$-classes or, except in a singular case, when $\Phi$ is induced by an isomorphism between the partial automorphism semigroups of $S$ and $T$.

It has long been known that $\phi_{E}$ extends to a bijection $\phi: E_{S} \cup N_{S} \rightarrow E_{T} \cup N_{T}$, where $N_{S}$ denotes the set of elements that belong to no subgroup of $S$. This is termed the "partial base bijection" in [14]. In the aperiodic (or "combinatorial") case where, by definition, all subgroups are trivial, $\phi$ is then a bijection between $S$ and $T$. In turn, $\phi$ induces $\Phi$ in the obvious way. It was shown by Ershova (see [14]) that as long as $S$ has no nontrivial isolated subgroups, then the partial bijection $\phi$ can be extended to a "base bijection", again denoted $\phi$, between $S$ and $T$. A priori, $\phi$ may extend non-uniquely.

In this paper, we study the properties of the partial base bijection (in $\S 1$ ) and of base bijections ( 22$)$ under a finiteness hypothesis termed quasi-connectedness, to be defined shortly. We thereby show that under this hypothesis (together with the hypothesis on $\phi_{E}$ and that on subgroups), if $S$ either (a) is fundamental or (b) is completely semisimple with at least three idempotents in each $\mathcal{D}$-class, any partial base bijection, as above, is necessarily an isomorphism. We then prove that the "quasi-archimedean" condition considered by the author in [11] and the "tightly connected" condition considered by Goberstein in [5] each implies quasi-connectedness, thereby deducing a main result of each of the cited papers. 
The property of quasi-connectedness is defined in two stages. Whilst somewhat technical, we will show in the last two sections of this paper how it relates to various finiteness conditions that have previously been developed in the context of lattice isomorphisms. As numerous examples have demonstrated, without some finiteness condition there may be insufficient linkage between subgroups within $\mathcal{D}$-classes, or between the $\mathcal{D}$-classes themselves, to ensure compatibility of the definition of $\phi$ between them.

Definition. Let $S$ be an inverse semigroup. If $a, b \in S$ with $b<a$ then $b b^{-1}<a a^{-1}$ and $b^{-1} b<a^{-1} a$. We shall say that $b$ is quasi-covered by $a$ if either $b b^{-1} \nless a^{2} a^{-2}$ or $b^{-1} b \nless a^{-2} a^{2}$. Observe that, of necessity, in this event $a \in N_{S}$ (for otherwise $a a^{-1}=a^{2} a^{-2}$ and dually).

Call $S$ quasi-connected if (i) $E_{S} \cup N_{S}$ is an order ideal of $S$ (under the natural partial order) and (ii) whenever $a, b \in N_{S}$ with $b<a$ then there is a sequence $b=b_{0}<b_{1}<\cdots<b_{n}=a$ in $S$ such that $b_{k-1}$ is quasi-covered by $b_{k}$, for each $k=1, \ldots, n$.

We remarked earlier that barring nontrivial isolated subgroups allows us to tie subgroups to the remainder of the semigroup (and to define the base bijections). The existence of nontrivial isolated subgroups does not preclude positive outcomes, however. In [8] it was shown that the free product of two groups, in the variety of inverse semigroups, is determined by its lattice of inverse subsemigroups, despite the isolation of the original groups within the free product. (In fact, [9], any non-trivial free product of inverse semigroups is determined by its lattice of inverse subsemigroups.) Another, simpler, result $\left[2\right.$, p.408], [10, Theorem 5.4] is that any Munn semigroup $T_{E}$ is lattice-determined whenever the semilattice $E$ has the property that each of its principal ideals is finite.

We conclude these preliminaries with some formal definitions and a reminder of some basic tools that will be used in the sequel.

The lattice $\mathcal{L}(S)$ of inverse subsemigroups of an inverse semigroup $S$ has as its zero the empty inverse subsemigroup. Note that for a group $G$, the empty inverse subsemigroup must be adjoined to obtain $\mathcal{L}(G)$. If $U, V \in \mathcal{L}(S)$, their join is denoted $U \vee V$ and is the same as their join as subsemigroups. If $A \subseteq S$, we denote by $\langle A\rangle$ the inverse subsemigroup that it generates.

The idempotents of an inverse semigroup $S$ form a semilattice, denoted $E_{S}$. The natural partial order on $S$ is defined by $a \leq b$ if $a=a a^{-1} b$; various useful equivalent properties may be found in [13], along with such basic semigroup concepts as Green's relations, ideals and principal factors. The notation $a \| b$ means that $a$ and $b$ are incomparable with respect to that order. A subset $A$ of a $S$ is an order ideal if $a \in A, b \in S, b<a$ imply $b \in A$.

The semigroup $S$ is aperiodic, or combinatorial, if all of its subgroups are trivial; it is fundamental if it possesses no nontrivial idempotent-separating congruences (so that, in particular, aperiodic inverse semigroups are fundamental); $S$ is completely semisimple if each principal factor is completely 0-simple or is a group (equivalently, $S$ contains no bicyclic subsemigroup - see below for the definition of the latter); $S$ is E-unitary if whenever $a \geq e \in E_{S}$ then $a \in E_{S}$.

Let $\Phi: \mathcal{L}(S) \rightarrow \mathcal{L}(T)$ be an isomorphism. Since $E_{S} \Phi=E_{T}, \Phi$ restricts to an isomorphism $\mathcal{L}\left(E_{S}\right) \rightarrow$ $\mathcal{L}\left(E_{T}\right)$, which induces a bijection $\phi_{E}: E \rightarrow F$ by the rule $\left\langle e \phi_{E}\right\rangle=\langle e\rangle \Phi, e \in E$. The bijection $\phi_{E}$ is characterized by the weak isomorphism property: if $e, f \in E$ then $e \| f$ if and only if $e \phi_{E} \| f \phi_{E}$, in which case $(e f) \phi_{E}=e \phi_{E} f \phi_{E}$.

Note that the class of groups is closed under $\mathcal{L}$-isomorphisms, since groups are characterized among inverse semigroups by the property that their semilattices of idempotents are trivial. Hence if $e \in E_{S}$ then $\Phi$ restricts to an $\mathcal{L}$-isomorphism between the maximal subgroups $H_{e}$ and $H_{e \phi_{E}}$. In particular, if $S$ is aperiodic, so is $T$.

Finally, we briefly review properties of monogenic inverse semigroups. According to [13, Theorem IX.3.11], each such semigroup is defined by exactly one of the following relations, where $k, l$ are positive integers: (i) $a^{k}=a^{-1} a^{k+1}$; (ii) $a^{k} a^{-1}=a^{-1} a^{k}$; (iii) $a^{k}=a^{k+l}$; (iv) $a=a$. Those semigroups determined 
by (i) possess a bicyclic kernel (least ideal); those determined by (ii) have an infinite cyclic group kernel; those determined by (iii) have a finite cyclic group kernel; that determined by (iv) is free. In the first three cases, if $k=1$ then the semigroup itself is bicyclic, infinite cyclic or finite cyclic, respectively. If $k \geq 2$, then it is an extension of its kernel by the quotient of the free monogenic inverse semigroup modulo the ideal generated by $a^{k}$ (the quotient then being in class (iii), with trivial kernel).

\section{The partial base bijection}

In this section we review the definition and basic properties of this map and prove the key technical results needed in the proofs of our main theorems.

As noted earlier, $N_{S}$ denotes the set of elements of $S$ that do not belong to a subgroup. For each $a \in N_{S}$, by [8] there is a unique element $b$ of $T$ such that $\langle a\rangle \Phi=\langle b\rangle,\left(a a^{-1}\right) \phi_{E}=b b^{-1}$ and $\left(a^{-1} a\right) \phi_{E}=b^{-1} b$. Setting $b=a \phi_{N}$ yields a bijection $\phi_{N}: N_{S} \rightarrow N_{T}$. Let $\phi=\phi_{E} \cup \phi_{N}$. We follow [14] in terming this the 'base partial bijection'. If $S$ is aperiodic, $\phi$ becomes the 'base bijection' of $S$ upon $T$, and then induces $\Phi$.

RESULT 1.1 Let $\Phi$ be an $\mathcal{L}$-isomorphism between inverse semigroups $S$ and $T$. There is a unique bijection $\phi: E_{S} \cup N_{S} \rightarrow E_{T} \cup N_{T}$ such that $\phi$ restricts to a weak isomorphism from $E_{S}$ to $E_{T},\langle a\rangle \Phi=\langle a \phi\rangle$ for all $a \in E_{S} \cup N_{S}$, and $\phi$ is $\mathcal{L}$ - and $\mathcal{R}$-preserving.

Properties of lattice isomorphisms of monogenic inverse semigroups are summarized in [14, §42]. If $S$ is such a semigroup and $\Phi$ a lattice isomorphism of $S$ upon an inverse semigroup $T$, then for all $a, b \in E_{S} \cup N_{S}$ such that $a b \in E_{S} \cup N_{S}$, then $(a b) \phi=a \phi b \phi$. In particular $\phi_{E}$ is always an isomorphism; and $\phi$ itself is an isomorphism if $S$ is aperiodic.

Throughout the rest of this section, the notation is that of Result 1.1. Assume that $\phi$ restricts to an isomorphism on $E_{S}$. We begin with a quite general tool.

PROPOSITION 1.2 Suppose $a \in N_{S}, e \in E_{S}$ and $e<a$. Then $e \phi<a \phi$.

Proof. Since $e<a, e a=a e=a$, whence $a^{-1} e=e a^{-1}=a$. Hence $w e=e w=e$ for all $w \in\langle a\rangle$, so that $\langle e, a\rangle=\langle a\rangle \cup\{e\}$. Now if $\langle a\rangle$ contains $e$, it must be aperiodic, since $e$ is its zero. Then, as noted in Section $1, \phi$ is an isomorphism on $\langle a\rangle$ and so $e \phi<a \phi$.

Otherwise $\langle e, a\rangle$ is the disjoint union of $\langle a\rangle$ and $\{e\}$. In that event, $\langle e \phi, a \phi\rangle$ is the disjoint union of $\langle a \phi\rangle$ and $\{e \phi\}$. Suppose $e \phi a \phi \in\langle a \phi\rangle$. Then $e \phi \geq(e \phi)(a \phi)(a \phi)^{-1}=(e \phi a \phi)(e \phi a \phi)^{-1} \in E_{\langle a \phi\rangle}$.

But from the first two sentences of the proof, $e \leq f$ for every $f \in E_{\langle a\rangle}$ and then, by hypothesis, $e \phi \leq f \phi$ for all such $f$. Since $e \phi$ was assumed not to belong to $\langle a \phi\rangle$, a contradiction is obtained.

The second key technical tool is the following, which extends [5, Lemma 2.4] and draws on techniques in the proofs of that lemma and of [11, Lemma 4.2] for its proof.

LEMMA 1.3 If $a, b \in N_{S}, b<a$ and $b$ is quasi-covered by $a$, then $b \phi<a \phi$.

Proof. To prove the first statement, we may assume that $b b^{-1} \nless a^{2} a^{-2}$, the other case being dual. Note first that if $b b^{-1}=a^{2} a^{-2}$ then $b=\left(a^{2} a^{-2}\right) a \in\langle a\rangle$ and since $b \in N_{S}$, it does not lie in the kernel of $\langle a\rangle$, if one exists. Thus by the remarks on monogenic inverse semigroups in Section 1 , $b \phi=\left(a^{2} a^{-2} a\right) \phi=(a \phi)^{2}(a \phi)^{-2}(a \phi)<a \phi$. So we may assume from now on that $b b^{-1} \not \leq a^{2} a^{-2}$.

Now by Result $1.1\langle a \phi\rangle=\langle a\rangle \Phi$ and $\langle b \phi\rangle=\langle b\rangle \Phi$. Since $b=\left(b b^{-1}\right) a \in E_{S} \vee\langle a\rangle, b \phi \in\langle b\rangle \Phi \subseteq$

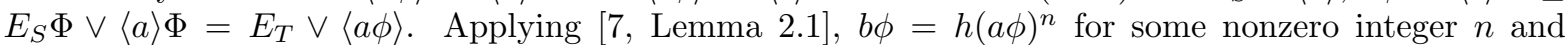


some $h \in E_{T}$. Thus $b \phi \leq(a \phi)^{n}$ and so in fact $b \phi=(b \phi)(b \phi)^{-1}(a \phi)^{n}$. Note that since $\phi$ preserves $\mathcal{R}$, $(b \phi)(b \phi)^{-1}=\left(b b^{-1}\right) \phi$. Thus $\left(b b^{-1}\right) \phi \leq(a \phi)^{n}(a \phi)^{-n}$.

Suppose $n>1$. Then $(a \phi)^{n}(a \phi)^{-n} \leq(a \phi)^{2}(a \phi)^{-2}=\left(a^{2} a^{-2}\right) \phi$, as above. Since $\phi_{E}$ is an isomorphism, $b b^{-1} \leq a^{2} a^{-2}$, a contradiction.

Next suppose $n<0$. Then $(b \phi)^{-1}(b \phi)=(a \phi)^{-n}\left(b b^{-1}\right) \phi(a \phi)^{n} \leq(a \phi)^{-n}(a \phi)^{n} \leq(a \phi)(a \phi)^{-1}$, so that $\left(b^{-1} b\right) \phi<\left(a a^{-1}\right) \phi$ and $b^{-1} b<a a^{-1}$. Since $b<a$ and the partial order is compatible with multiplication and inversion, $b b^{-1}=b\left(b^{-1} b\right) b^{-1} \leq a^{2} a^{-2}$, once again a contradiction.

Thus $n=1$ and $b \phi<a \phi$.

COROLLARY 1.4 If $S$ is quasi-connected then $b \phi<a \phi$ whenever $b<a$ and $a \in E_{S} \cup N_{S}$.

Proof. According to Proposition 1.2 the conclusion holds when $b \in E_{S}$. Since $E_{S} \cup N_{S}$ is an order ideal, the remaining case to consider is when $b \in N_{S}$. Then there is a sequence $b=b_{0}<b_{1}<\cdots<b_{n}=a$ such that $b_{k-1} \in N_{S}$ and $b_{k-1}$ is quasi-covered by $b_{k}$, for each $k=1, \ldots, n$. Iteration of the previous lemma yields the requisite inequality.

\section{Base bijections}

In the aperiodic case, the partial base bijection $\phi: E_{S} \cup N_{S} \rightarrow E_{T} \cup N_{T}$ is actually a bijection. We may extend $\phi$ to nontrivial non-isolated subgroups as follows. For each idempotent $e$ in such a subgroup of $S, D_{e}$ contains more than one idempotent. Choose and fix an element $r_{e}$ of $N_{S} \cap R_{e}$. For $a \in H_{e}$ define $a \phi=\left(r_{e} \phi\right)(s \phi)^{-1}$, where $s=a^{-1} r_{e}$ is the unique element of $H_{r_{e}}$ such that $a=r_{e} s^{-1}$.

Thus in general $\phi$ may be extended to any individual $\mathcal{D}$-class that is not simply a group. Then (Ershova: see $[14, \S 43.7]$ ) as long as $S$ contains no nontrivial isolated subgroups, this rule defines a base bijection $\phi$ from $S$ to $T$. Observe that, a priori, there is no reason that $\phi$ should be uniquely defined, since the elements $r_{e}$ may be chosen arbitrarily. The use of the term "the base bijection" in the literature is therefore misleading. Note that in [14] and [11], $\phi$ was denoted $\theta$; in [5] it was denoted $\hat{\phi}$.

RESULT 2.1 [14, §43.7] Let $\Phi$ be an $\mathcal{L}$-isomorphism between inverse semigroups $S$ and $T$, where $S$ (and therefore T) has no nontrivial isolated subgroups. Any base bijection $\phi: S \rightarrow T$, as just defined, extends the partial base bijection $\phi: E_{S} \cup N_{S} \rightarrow E_{T} \cup N_{T}$ and thereby retains the properties exhibited in Result 1.1; in particular, it preserves $\mathcal{L}$ and $\mathcal{R}$. If $\Phi$ is induced by an isomorphism $\kappa: S \rightarrow T$, then $\kappa=\phi$ (and so in that case the base bijection is uniquely defined).

It is not known (see $[14, \S 43.7]$ ) whether the base bijection $\phi$ always induces $\Phi$, even should it be an isomorphism. It is known $[14,44.5]$ that $\phi$ induces $\Phi$ in the case of Brandt semigroups (that are not groups with adjoined zero).

In order to extend the properties of the partial base bijection found in the previous section, in particular Corollary 1.4, we state two results that are extracted from deep within key proofs of the main theorems that we are generalizing in this paper. We state them in the greatest generality we can, for possible future use.

Prior to the first of these, we need to link the outcome of Corollary 1.4 to the hypothesis relevant to fundamental inverse semigroups in $\S 3$.

RESULT 2.2 [5, Lemma 1.2] Let $S$ and $T$ be inverse semigroups and $\phi: S \rightarrow T$ be a bijection that preserves $\mathcal{L}$ and $\mathcal{R}$ and restricts to an isomorphism on $E_{S}$. If $b<a$ implies $b \phi<$ a $\phi$ for all $a \in N_{S}$, then $\left(a^{-1} e a\right) \phi=(a \phi)^{-1}(e \phi)(a \phi)$ for all $a \in N_{S}, e \in E_{S}$. 
The connection between the properties in this result is more clearly seen if one verifies that (1) the assumption is equivalent to the statement that for all $a \in N_{S},(e a) \phi=(e \phi)(a \phi)$ for all $e<a a^{-1}$; and (2) the conclusion is equivalent to the statement that for all $a \in N_{S},(e a) \phi \mathcal{H}(e \phi)(a \phi)$ for all $e<a a^{-1}$.

The next result was the essential ingredient in the proof of the theorem of Goberstein [5, Theorem $2.5]$ that we will generalize in $\S 3$. We include Goberstein's proof so that it is clear what hypotheses are required in its derivation.

RESULT 2.3 Let $S$ be an inverse semigroup with no nontrivial isolated subgroups, and let $\Phi$ be a lattice isomorphism of $S$ upon T, inducing a base bijection $\phi: S \rightarrow T$. Suppose that $\phi$ restricts to an isomorphism on $E_{S}$. If the equation $\left(a^{-1} e a\right) \phi=(a \phi)^{-1}(e \phi)(a \phi)$ holds for all $a \in N_{S}, e \in E_{S}$, then it holds for all $a \in S, e \in E_{S}$.

Proof. By hypothesis, it remains to treat the situation in which a lies in a subgroup. Put $f=a a^{-1}=a^{-1} a$. Then $a \phi=\left(r_{f} \phi\right)(s \phi)^{-1}$, where $r_{f} \in N_{S} \cap R_{f}$ and $a=r_{f} s^{-1}$. Thus $\left(a^{-1} e a\right) \phi=$ $\left(s\left(r_{f}{ }^{-1} e r_{f}\right) s^{-1}\right) \phi=(s \phi)\left(r_{f}^{-1} e r_{f}\right) \phi(s \phi)^{-1}$, applying the hypothesis to $s^{-1} \in N_{S}$ and $r_{f}^{-1} e r_{f} \in E_{S}$; and $(s \phi)\left(r_{f}^{-1} e r_{f}\right) \phi(s \phi)^{-1}=(s \phi)\left(\left(r_{f} \phi\right)^{-1}(e \phi)\left(r_{f} \phi\right)\right)(s \phi)^{-1}=(a \phi)^{-1}(e \phi)(a \phi)$, applying it to $r_{f} \in N_{S}$ and $e \in E_{S}$.

Next we consider an analogous result relevant to completely semisimple inverse semigroups, the essence of which is embedded in the proof of [11, Theorem 4.5]. This result uses only the properties of base bijections, not the definition. Again, we include a proof in order to clarify the precise hypotheses required in its derivation.

A product $a b$ (sometimes denoted $a \cdot b$ for clarity) is restricted if $a^{-1} a=b b^{-1}$; in that event $a b \in$ $R_{a} \cap L_{b}$. The relevance of this property owes much to the fact (e.g. [12, Theorem 3.1.5]) that if a mapping between two inverse semigroups is order preserving, restricts to a homomorphism on $E_{S}$ and preserves restricted products, then it is a homomorphism.

LEMMA 2.4 Let $S$ be an inverse semigroup with no nontrivial isolated subgroups and suppose $\phi: S \rightarrow$ $T$ is a mapping of inverse semigroups that restricts to a homomorphism on $E_{S}$, preserves restricted products and satisfies $b \phi \leq a \phi$ whenever $a, b \in S, b<a$ and $a \in E_{S} \cup N_{S}$. Then $\phi$ is order preserving and hence a homomorphism.

Proof. It remains to verify that $b \phi \leq a \phi$ whenever $b<a$ and $a \in H_{f}$, say, $f \in E_{S}$. By hypothesis, there exists an idempotent $g$, say, in $D_{a}$, distinct from $f$. If $r \in R_{f} \cap L_{g}$, then $a=r s$, where $s=r^{-1} a \in$ $L_{f} \cap R_{g}$ and the product is restricted.

Now put $e=b b^{-1}, d=b^{-1} b$ : then since $b<a, b=e a=a d$. Thus $b=e b d=e a d=(e r)(s d)$, where $e r \in R_{e}$ and $s d \in L_{d}$. Also, $(r s) d s^{-1} r^{-1}=a d a^{-1}=(a d)(a d)^{-1}=e=f e f=\left(r r^{-1}\right) e r r^{-1}$ and then, since $r^{-1} r s=s$, conjugation by $r$ yields $s d s^{-1}=r^{-1} e r$, so the product $e r \cdot s d$ is restricted. Applying the hypothesis that $\phi$ preserves restricted products, $b \phi=(e r) \phi(s d) \phi$. Further, er $<r \in N_{S}$, so $(e r) \phi \leq r \phi$; similarly $(s d) \phi \leq s \phi$. Hence $b \phi \leq r \phi s \phi=a \phi$, once again applying the hypothesis on restricted products.

Although the material in the remainder of this section does not find direct use in the sequel, in light of the previous lemma it is appropriate to include at this point. Ershova (see [14, 44.6]) proved that in the case of Brandt semigroups with at least three nonzero idempotents, any base bijection $\phi$ is an isomorphism. We shall rederive that theorem below as a consequence of a more general analysis of properties of $\phi$. Our results will apply, for instance, to simple inverse semigroups that are not 0-simple.

In the following, once more $\Phi$ is a lattice isomorphism between inverse semigroups $S$ and $T$, inducing both the partial base bijection $\phi$ and its extension to all $\mathcal{D}$-classes that are not just groups. 
Let us call a restricted product super-restricted if none of $a, b, a b$ lies in a subgroup. We show that whenever a $\mathcal{D}$-class contains more than two idempotents, to prove a base bijection $\phi$ preserves restricted products on $D$ it suffices to show only that $\phi$ preserves super-restricted products on $D$. Note that this reduces the problem to one involving the partial base bijection. Along the way, we also show that $\phi$ is uniquely defined in this situation and that that property alone has useful consequences.

LEMMA 2.5 Suppose the $\mathcal{D}$-class $D$ contains at least three idempotents and $\phi$ preserves super-restricted products on $D$. Then $\phi$ is uniquely defined on $D$, that is, for each idempotent e in $D$, the definition of $\phi$ on $H_{e}$ is independent of the choice of $r_{e}$.

Proof. Let $f, g$ be distinct idempotents of $D$, different from $e$. Let $r_{e} \in R_{e} \cap L_{f}, s_{e} \in R_{e} \cap L_{g}$. Then for any $a \in H_{e}, a=r_{e} t^{-1}=s_{e} u^{-1}$, where $t=a^{-1} r_{e} \in R_{e} \cap L_{f}$ and $u=a^{-1} s_{e} \in R_{e} \cap L_{g}$. It follows that $s_{e}=r_{e} t^{-1} u$.

Using $r_{e}$, we may define $a \phi_{1}=\left(r_{e} \phi\right)(t \phi)^{-1}$; using $s_{e}$, we may define $a \phi_{2}=\left(s_{e} \phi\right)(u \phi)^{-1}$. We will show that $a \phi_{1}=a \phi_{2}$. Since the choices of $r_{e}$ and $s_{e}$ were arbitrary within their $\mathcal{H}$-classes, this also proves that the definition of $a \phi_{1}$ is independent of the choice of $r_{e}$.

Now $t^{-1} u \in R_{f} \cap L_{g}$, so the product $t^{-1} \cdot u$ is super-restricted and $\left(t^{-1} u\right) \phi=(t \phi)^{-1} u \phi$, by hypothesis. Likewise, the product $r_{e} \cdot t^{-1} u$ is super-restricted, the result lying in $R_{e} \cap L_{g}$, so $s_{e} \phi=r_{e} \phi\left(t^{-1} u\right) \phi=$ $r_{e} \phi(t \phi)^{-1} u \phi=a \phi_{1} u \phi$ and $a \phi_{2}=s_{e} \phi(u \phi)^{-1}=a \phi_{1} u \phi(u \phi)^{-1}=a \phi_{1}\left(u u^{-1}\right) \phi=a \phi_{1} e \phi=a \phi_{1}$.

LEMMA 2.6 For any $\mathcal{D}$-class with at least two idempotents, if $\phi$ is known to be uniquely defined on $D$, then it preserves all restricted products $a \cdot b$ on $D$ that are not super-restricted.

Hence if $D$ contains at least three idempotents, then if $\phi$ preserves all super-restricted products on $D$, it preserves all restricted products on $D$.

Proof. There are essentially three cases to consider. Each follows easily by making a judicious choice of " $r$ " in the definition of $\phi$ on $H_{e}$. Let $a \cdot b$ be a restricted product in $D$.

(I) Both $a, b \in N_{S}$ but $a b \notin N_{S}$.

Say $a \in R_{e} \cap L_{f}$, so $b \in R_{f} \cap L_{e}$ and $a b \in H_{e}$. Now by hypothesis, we may set $r_{e}=a$ in order to define $\phi$ on $H_{e}$. Then in the definition of $(a b) \phi, s=b^{-1}$, whence $(a b) \phi=(a \phi)\left(b^{-1} \phi\right)^{-1}=a \phi b \phi$.

(II) Exactly one of $a, b$ lies in $N_{S}$ (whence necessarily $a b \in N_{S}$ ).

We may suppose that $a \in H_{e}$ and $b \in R_{e} \cap L_{f}$, say, $f \neq e$, so that $a b \in H_{b}$. The other case is dual. But now the product $(a b) \cdot b^{-1}$ falls under case (I), since $(a b) b^{-1}=a$, so $a \phi=(a b) \phi(b \phi)^{-1}$ and $a \phi b \phi=(a b) \phi$.

(III) Neither $a$ nor $b$ lies in $N_{S}$ (whence necessarily $a b \notin N_{S}$ ).

We may suppose that $a, b, a b \in H_{e}$. Let $f$ be an idempotent of $D$ distinct from $e$ and choose $r_{e} \in R_{e} \cap L_{f}$. Then $a=r_{e} s^{-1}$, for some $s \in R_{e} \cap L_{f}$, and so $a b=r_{e} u^{-1}$, where $u=b^{-1} s \in R_{e} \cap L_{f}$. Hence $(a b) \phi=\left(r_{e} \phi\right)\left(\left(b^{-1} s\right) \phi\right)^{-1}=\left(r_{e} \phi\right)\left(s^{-1} b\right) \phi$ (since $\phi$ preserves inverses on $N_{S}$ ). But the product $s^{-1} \cdot b$ falls under case (II) and so $\left(s^{-1} b\right) \phi=(s \phi)^{-1} b \phi$ and $(a b) \phi=a \phi b \phi$, using the definition of $a \phi$.

COROLLARY 2.7 [14, 44.6] For any Brandt semigroup with more than two nonzero idempotents, the base bijection $\phi$ is an isomorphism (and so uniquely defined).

Proof. According to the above, only preservation of super-restricted products $a \cdot b$ remains to be verified. But for such $a, b$, it is easily seen that $\langle a, b\rangle \cap R_{a} \cap L_{b}=\{a b\}$ (or see the reference in [14]) and similarly for the image under $\Phi$. 
For Brandt semigroups with exactly two nonzero idempotents it is apparently unknown whether this remains true (see [15, Problem 7.11]). The above results are relevant to any simple inverse semigroup that is not a group. However lattice determinability for such semigroups is little understood outside the fundamental case.

\section{Fundamental inverse semigroups and the hypotheses of Gob- erstein}

Fundamental inverse semigroups were defined in the introduction. They provide a way of tying isolated subgroups into the larger structure of the semigroup. It is well known (see [13], Theorem V.3.2 and formula (V.3.3)) that an inverse semigroup $S$ is fundamental if and only if for every $x, y \in S$, whenever $x^{-1} e x=y^{-1} e y$ for all $e \in E_{S}$, then $x=y$.

In our context, the key to the usefulness of fundamentality is Goberstein's general lemma, adapted in [5] from [3, Lemma 2.1].

RESULT 3.1 [5, Result 1.1] Let $S$ and $T$ be inverse semigroups and $\phi: S \rightarrow T$ a bijection that restricts to an isomorphism on $E_{S}$. If either $S$ or $T$ is fundamental, then $\phi$ is an isomorphism if and only if $\left(a^{-1} e a\right) \phi=(a \phi)^{-1}(e \phi)(a \phi)$ for all $a \in S, e \in E_{S}$.

Similarly to the remark in the previous section, the second of the equivalent statements is itself equivalent to the statement that for all $a \in S,(e a) \phi \mathcal{H}(e \phi)(a \phi)$, for all $e<a a^{-1}$.

THEOREM 3.2 Let $S$ be a fundamental inverse semigroup with no nontrivial isolated subgroups, and $\Phi: \mathcal{L}(S) \rightarrow \mathcal{L}(T)$ a lattice isomorphism for some inverse semigroup $T$, inducing the base bijection $\phi: S \rightarrow T$. Suppose that $S$ is quasi-connected and $\phi_{E}$ is an isomorphism. Then $\phi$ is an isomorphism.

Proof. This is now immediate from Corollary 1.4, Result 2.2, Result 2.3 and Result 3.1.

As remarked in [5], in the aperiodic or completely semisimple case $\phi$ actually induces $\Phi$ and is the unique isomorphism to do so.

In the remainder of this section we examine two hypotheses considered by Goberstein in earlier papers and show that tight connectedness, the main hypothesis in [5], implies quasi-connectedness. We thereby deduce as a corollary [5, Theorem 2.5].

Goberstein introduced the following notion in [2]. Let $S$ be an inverse semigroup. If $a \in S, e \in$ $E_{S}, e<a a^{-1}$ and there is no $f \in E_{\langle a\rangle}$ such that $e<f<a a^{-1}$, we say that $e$ is $a$-covered by $a a^{-1}$, written $e \prec_{a} a a^{-1}$. If $e<a a^{-1}$ and there is a sequence of idempotents $e=e_{0}<e_{1}<\cdots<e_{n}=a a^{-1}$ for which $e_{k-1}$ is $e_{k} a$-covered by $e_{k}$ for $k=1, \ldots, n$, then $\left(e_{0}, e_{1}, \ldots, e_{n}\right)$ is called a short bypass from $e$ to $a a^{-1}$. If such a short bypass exists for every such $e$ and $a$, then $S$ is said to be shortly connected.

LEMMA 3.3 Let $S$ be an inverse semigroup and suppose $a, b \in S, b<a, a \in N_{S}$. If $b b^{-1}$ is a-covered by $a a^{-1}$, then $b$ is quasi-covered by $a$.

Proof. Suppose both $b b^{-1}<a^{2} a^{-2}$ and $b^{-1} b<a^{-2} a^{2}$ hold. Since $a^{2} a^{-2} \leq a a^{-1}$, then the $a$-covering property implies $a^{2} a^{-2}=a a^{-1}$, from which it follows that $a a^{-1} \geq a^{-1} a$. In fact, the inequality is strict, since $a \in N_{S}$.

From $b^{-1} b<a^{-2} a^{2}$ we obtain $b b^{-1}=b\left(b^{-1} b\right) b^{-1} \leq b\left(a^{-2} a^{2}\right) b^{-1}$. Since $b<a$ and the partial order is compatible with multiplication and inversion, $b\left(a^{-2} a^{2}\right) b^{-1} \leq a\left(a^{-2} a^{2}\right) a^{-1}=\left(a a^{-1}\right)\left(a^{-1} a\right)=a^{-1} a<$ $a a^{-1}$, using the final inequality and remark of the previous paragraph. By hypothesis, $b b^{-1}=a^{-1} a$, 
whence $b=b b^{-1} a=a^{-1} a^{2}$. However, in that event $b^{-1} b=a^{-2} a^{2}$, contradicting the strictness of the original inequality.

COROLLARY 3.4 If $S$ is a shortly connected inverse semigroup in which $E_{S} \cup N_{S}$ is an order ideal, then $S$ is quasi-connected.

Proof. Suppose $S$ is shortly connected and $a, b \in N_{S}, b<a$. Let $e=b b^{-1}$, so that $e<a a^{-1}$. Let $\left(e_{0}, e_{1}, \ldots, e_{n}\right)$ be a short bypass from $e$ to $a a^{-1}$ and for $k=0, \ldots, n$ put $b_{k}=e_{k} a$, so that $b_{k} b_{k}^{-1}=e_{k}$ and $b=b_{0}<b_{1}<\ldots<b_{n}=a$. Since $E_{S} \cup N_{S}$ is an order ideal and $b \notin E_{S}$, each $b_{k} \in N_{S}$. Now repeated application of the previous lemma yields the desired conclusion.

In [5], Goberstein strengthened short connectedness as follows. If $e<a a^{-1}$ then $e$ is tightly a-covered by $a a^{-1}$ if $e \prec_{a} a a^{-1}$ and both $a$ and $e a$ belong to $E_{S} \cup N_{S}$. The semigroup $S$ is said to be tightly connected if for every $e \in E_{S}$ and every $a \in E_{S} \cup N_{S}$ such that $e<a a^{-1}$, there is a tight bypass from $e$ to $a a^{-1}$, namely a short bypass for which (in the above notation) $e_{k-1}$ is tightly $e_{k} a$-covered by $e_{k}$ for $k=1, \ldots, n$.

Clearly, for aperiodic inverse semigroups, the two definitions coincide. The precise relationship between them is given by the following.

PROPOSITION 3.5 An inverse semigroup $S$ is tightly connected if and only if $S$ is shortly connected and $E_{S} \cup N_{S}$ is an order ideal. It follows from Corollary 3.4 that every tightly connected inverse semigroup is quasi-connected.

Proof. Suppose $S$ is tightly connected. We first show that $E_{S} \cup N_{S}$ is an order ideal. Let $a \in E_{S} \cup N_{S}$ and suppose $b \in S, b<a$. Clearly, if $a \in E_{S}$ then $b \in E_{S}$. So assume $a \in N_{S}$. Then $e=b b^{-1}<a a^{-1}$ and by hypothesis there is a tight bypass $e=e_{0}<e_{1}<\cdots<e_{n}=a a^{-1}$ with, by definition, each $e_{k} a \in E_{S} \cup N_{S}$. Hence $b=e_{0} a$ has this property, as required.

Now to show $S$ is shortly connected, take arbitrary $a \in S, e \in E_{S}$ with $e<a a^{-1}$. If $a$ belongs to a subgroup of $S$ then $E_{\langle a\rangle}=\left\{a a^{-1}\right\}$, whence $e<a a^{-1}$ is a short bypass from $e$ to $a a^{-1}$. Otherwise $a \in E_{S} \cup N_{S}$ and, by definition there is once again a short bypass from $e$ to $a a^{-1}$.

To prove the converse, suppose $a \in E_{S} \cup N_{S}, e \in E_{S}, e<a a^{-1}$. Then there is a short bypass $e=e_{0}<e_{1} \cdots<e_{n}=a a^{-1}$. Now for each $k, e_{k} a<a$ so, by hypothesis $e_{k} a \in E_{S} \cup N_{S}$, that is, the bypass is in fact tight.

As a consequence of the final statement of this proposition, Theorem 3.2 specializes to [5, Theorem $2.5]$.

Quasi-connectedness is prima facie a weaker property than tight connectedness in two ways. First, the latter property requires the existence of a short bypass from any idempotent $e$ strictly less than $a a^{-1}$, whereas the former property only requires (ii) of its definition to hold when $e a \in N_{S}$ (not when $\left.e a \in E_{S}\right)$. For instance, adjoining a zero to a free monogenic inverse semigroup $\langle a\rangle$ yields an inverse semigroup that is quasi-connected (since $\langle a\rangle$ is so, as we shall see in the next section) but is not tightly (equivalently shortly) connected, since there is no short bypass from 0 to $a a^{-1}$.

The next proposition illustrates a second way in which quasi-connectedness is weaker than tight connectedness. Recall that an inverse semigroup is E-unitary if whenever $e \in E_{S}, a \in S$ and $e<a$ then $a \in E_{S}$. Under this hypothesis, the situation in the previous paragraph cannot occur.

PROPOSITION 3.6 There is an E-unitary, aperiodic inverse semigroup that is quasi-connected but not shortly (equivalently tightly) connected, and in which the converse of Lemma 3.3 fails. 
Proof. Let $A=\langle a\rangle$ be a free monogenic inverse semigroup and $B=\langle b\rangle$ be a bicyclic semigroup, with $b b^{-1}>b^{-1} b$. Let $S$ be the retract ideal extension of $B$ by $A$ defined by the homomorphism $A \rightarrow B$ that is induced by $a \rightarrow b$.

Multiplication of an element of $B$ by an element of $A$ is accomplished by substituting $b$ for $a$ in any expression for the element of $A$ in terms of its generator. Hence an idempotent $e$ of $B$ is below an idempotent $f$ of $A$ if and only if $e$ is below (not necessarily strictly) the idempotent of $B$ corresponding to $f$. Since $b b^{-1}=b^{n} b^{-n}$ for every $n>1$ and $b b^{-1}>b^{-1} b>b^{-2} b^{2}>\cdots$, the following statements are easily verified.

The elements $a, b$ belong to $N_{S}$ and satisfy $b<a, b^{-1} b<a^{-1} a$ but $b^{-1} b \nless a^{-2} a^{2}$, so $b$ is quasi-covered by $a$; however $b b^{-1}<a a^{-1}$ and $b b^{-1}$ is not $a$-covered by $a a^{-1}$ since $b b^{-1}$ is below each of the distinct idempotents $a^{n} a^{-n}, n>1$, of $\langle a\rangle$. Hence the converse of Lemma 3.3 fails.

In fact since $b b^{-1} \nless a^{-1} a$, the only idempotents between $b b^{-1}$ and $a a^{-1}$ are those listed above. Similarly, then, $b b^{-1}$ is not $\left(a^{n} a^{-n}\right) a$-covered by $a^{n} a^{-n}$ for any $n>1$. Hence there is no short bypass from $b b^{-1}$ to $a a^{-1}$ and so $S$ fails to be shortly connected.

That $S$ is quasi-connected will follow from the results of the next section (see the remarks following Proposition 4.4).

Finally, we briefly mention a strengthening of the shortly connected property considered by Goberstein in [2], namely that of being "shortly linked", under which whenever $a \in S, e \in E_{S}$ and $e<a a^{-1}$, there is a short bypass from $e$ to $a a^{-1}$ in which every term but $e$ belongs to $\langle a\rangle$ itself. In light of the lemma above, we might define the term "tightly linked" by the conjunction of this property with the property that $E_{S} \cup N_{S}$ be an order ideal. In [4], Goberstein gave examples of shortly connected inverse semigroups that are not shortly linked.

\section{Completely semisimple inverse semigroups and earlier hy- potheses of the author}

Ershova's Result 2.7 was used by the author to prove the following.

RESULT 4.1 [11, Lemma 4.4] Let $S$ be a completely semisimple inverse semigroup with the property that each nonaperiodic $\mathcal{D}$-class contains at least three idempotents. If $\Phi$ is a lattice isomorphism from $S$ to an inverse semigroup $T$ then any base bijection $\phi$ preserves restricted products, that is, $(a b) \phi=a \phi b \phi$ for any restricted product $a \cdot b$.

THEOREM 4.2 Let $S$ be a completely semisimple inverse semigroup in which each nonaperiodic $\mathcal{D}$ class contains at least three idempotents, and $\Phi: \mathcal{L}(S) \rightarrow \mathcal{L}(T)$ a lattice isomorphism for some inverse semigroup $T$, inducing the base bijection $\phi$. Suppose that $S$ is quasi-connected and $\phi_{E}$ is an isomorphism. Then $\Phi$ is induced by a unique isomorphism, namely the base bijection $\phi$.

Proof. The hypotheses in the first sentence of the statement imply that $\phi$ induces $\Phi$, by [11, Lemma 2.4], and that $\phi$ preserves restricted products, by the result just stated. Then Corollary 1.4 and Lemma 2.4 combine to prove that $\phi$ is an isomorphism (and so the unique one that induces $\Phi$, by Result 2.1).

Without any finiteness assumption, the result may fail, as shown by [11, Example 4.7]. Also observe that if Ershova's theorem is indeed true for all Brandt semigroups (that are not groups with adjoined zero) the proof of the theorem would extend to all quasi-connected, completely semisimple inverse semigroups without nontrivial isolated subgroups. 
In the rest of this section we examine several hypotheses studied by the author and show that each implies quasi-connectedness. We thereby deduce [11, Theorems 4.3 and 4.5].

In [11, Proposition 3.1(1)], the author showed that an inverse semigroup is shortly linked (see the end of the previous section for the definition) if and only if no idempotent is strictly below infinitely many idempotents of any monogenic inverse subsemigroup. In the same paper, we considered several definitions that were motivated both by this property and by the archimedean property introduced in [7]: $E_{S}$ is archimedean in $S$ if whenever $a \in S, e \in E_{S}, e<a a^{-1}$ and $a a^{-1}>a^{-1} a$, then $a^{-n} a^{n} \leq e$ for some positive integer $n$.

An inverse semigroup $S$ is pseudo-archimedean if no idempotent of $S$ is strictly below every idempotent of a free monogenic or bicyclic inverse subsemigroup. Clearly, every shortly linked inverse semigroup is pseudo-archimedean. Example 4.11 of [1] shows that the pseudo-archimedean property is strictly weaker than that of being shortly linked, even in aperiodic, E-unitary, completely semisimple inverse semigroups. It is not difficult to see that this example is also not shortly connected, thus providing (in light of Proposition 4.4 below) a rather more complicated alternative to the example in Proposition 3.6 above.

In [11], the author introduced the following conditions. An inverse semigroup $S$ faintly archimedean if whenever an idempotent $e$ of $S$ is strictly below every idempotent of a bicyclic or free monogenic inverse subsemigroup $\langle a\rangle$, then $e<a$. Adjoining a zero to a free monogenic inverse semigroup yields a faintly archimedean inverse semigroup that is not pseudo-archimedean. It is straightforward to verify that the example constructed in Proposition 3.6 is faintly archimedean. The semigroup $S$ quasi-archimedean if whenever an idempotent $e$ is (not necessarily strictly) below every idempotent of $\langle a\rangle$, where $a \in N_{S}$, then $e<a$.

PROPOSITION 4.3 The following are equivalent for an inverse semigroup $S$ :

(1) $S$ is quasi-archimedean;

(2) if $a \in N_{S}, b<a$ and $b b^{-1}$ is below every idempotent of $\langle a\rangle$, then $b \in E_{S}$;

(3) $S$ is faintly archimedean and $\langle a\rangle$ is aperiodic for each $a \in N_{S}$;

(4) $S$ is faintly archimedean and $E_{S} \cup N_{S}$ is an order ideal of $S$;

(5) for each $a \in N_{S},\langle a\rangle$ is aperiodic and whenever $b<a, b b^{-1}<a^{n} a^{-n}$ and $b^{-1} b<a^{-n} a^{n}$ for every positive integer $n$, then $b \in E_{S}$.

Proof. All of these equivalences are proven in [11, Proposition 3.3] except (4). Suppose $S$ is quasiarchimedean, $a \in N_{S}$ and $b<a$. Put $e=b b^{-1}$. Now if $b \in H_{e}$ then $e=b^{n} b^{-n}<a^{n} a^{-n}$ for every nonzero integer $n$, and so $e$ is below every idempotent of $\langle a\rangle$. By (2), b=e. Hence $b \in E_{S} \cup N_{S}$.

To prove that (4) implies (3) we show that for any inverse semigroup $S$ in which $E_{S} \cup N_{S}$ is an order ideal, $\langle a\rangle$ is aperiodic for each $a \in N_{S}$. Refer to the classification of monogenic inverse semigroups in the introduction. The only nonaperiodic ones are those in class (ii) and those in class (iii) with $l>1$. In each case, if $a$ is a generator then the kernel is a group with identity $e=a^{n} a^{-n}$, containing the nonidempotent $e a<a$, contradicting the hypothesis.

PROPOSITION 4.4 Every quasi-archimedean inverse semigroup is quasi-connected.

Proof. We use (5) of the previous proposition. Suppose $a, b \in N_{S}, b<a$. Since $b \notin E_{S}$, either there exists a greatest positive integer $n$ such that $b b^{-1}<a^{n} a^{-n}$ or the left-right dual holds. By the 
self-dual nature of quasi-connectedness, we need only consider the former situation. For $1 \leq k \leq n$ put $c_{k}=\left(a^{k} a^{-k}\right) a$. Then $c_{k} c_{k}^{-1}=a^{k} a^{-k}$. Since $c_{k}^{2}=\left(a^{k} a^{-k}\right) a\left(a^{k} a^{-k}\right) a=a^{k+1} a^{-k} a$, it follows that $c_{k}^{2} c_{k}^{-2}=a^{k+1} a^{-k} a a^{-1} a^{k} a^{-(k+1)}=a^{k+1} a^{-k} a^{k} a^{-(k+1)}=a^{k+1} a^{-(k+1)}$.

Note that the idempotents $a^{k} a^{-k}, k=1, \ldots, n$, are distinct. For if $a^{k} a^{-k}=a^{k+m} a^{-(k+m)}$, with $m>0$, then $a^{k} a^{-k}=a^{l} a^{-l}$ for all $l>0$, leading to a contradiction to the assumption on $b b^{-1}$.

Then $b<c_{n}<c_{n-1}<\cdots<c_{1}=a$. Now $b b^{-1}<c_{n} c_{n}^{-1}$ and $c_{n}^{2} c_{n}^{-2}=a^{n+1} a^{-(n+1)}$. By the assumption on $b b^{-1}$, therefore $b b^{-1} \nless c_{n}^{2} c_{n}^{-2}$. Thus $b$ is quasi-covered by $c_{n}$. If $n=1$, the sequence $b<c_{1}=a$ suffices.

If $n>1$, then for $1 \leq k \leq n, c_{k} c_{k}^{-1}<c_{k-1} c_{k-1}^{-1}$ and $c_{k} c_{k}^{-1} \nless c_{k-1}^{2} c_{k-1}^{-2}$ (since equality holds, by the calculation above). That is, $c_{k}$ is quasi-covered by $c_{k-1}$.

Setting $b=b_{0}$ and $b_{k}=c_{n+1-k}$ therefore yields the requisite sequence of quasi-coverings in this case

As a consequence of the final statement of this proposition, Theorem 3.2 specializes to [11, Theorem 2.6] and Theorem 4.2 specializes to [11, Theorem 4.5].

It was observed above that the example in the proof of Proposition 3.6 is faintly archimedean. By virtue of Propositions 4.3 and 4.4, it is therefore quasi-connected.

In [6, Examples 5.5, 5.6], Goberstein provided aperiodic, shortly connected inverse semigroups that are not faintly archimedean. By virtue of Corollary 3.4, these examples also serve to distinguish quasiconnectedness from the quasi-archimedean property.

\section{References}

[1] K.H. Cheong and Peter R. Jones, Inverse semigroups determined by their lattices of convex inverse subsemigroups, II, Algebra Universalis 49 (2003), 81-106.

[2] Simon M. Goberstein, Inverse semigroups with isomorphic partial automorphism semigroups, J. Austral. Math. Soc. (Ser. A) 47 (1989), 399-417.

[3] S.M. Goberstein, Inverse semigroups determined by their bundles of correspondences, J. Algebra 125 (1989), 474-488.

[4] S.M. Goberstein, A note on shortly connected inverse semigroups, Bull. Austral. Math. Soc 43 (1991), 463-466.

[5] Simon M. Goberstein, Inverse semigroups determined by their partial automorphism monoids, J. Australian Math. Soc. (to appear).

[6] Simon M. Goberstein, $\mathcal{P} \mathcal{A}$-isomorphisms of inverse semigroups, Algebra Universalis (to appear).

[7] P.R. Jones, Distributive inverse semigroups, J. London Math. Soc. (2) 17 (1978), 457-466.

[8] P.R. Jones, Inverse semigroups determined by their lattices of inverse subsemigroups, J. Austral. Math. Soc. (Ser. A) 30 (1981), 321-346.

[9] P.R. Jones, Lattice isomorphisms of free products of inverse semigroups, J. Algebra 89 (1984), 293-318.

[10] Peter R. Jones, Inverse semigroups and their lattices of inverse subsemigroups, in "Lattices, Semigroups and Universal Algebra", ed. J. Almeida et al, Plenum Press, New York, 1990. 
[11] Peter R. Jones, On lattice isomorphisms of inverse semigroups, Glasgow Math. J. 46 (2004), 1932004.

[12] Mark V. Lawson, Inverse Semigroups, (World Scientific Publ. Co., Singapore (1998)).

[13] M. Petrich, Inverse Semigroups, (Wiley, New York, 1984).

[14] L.N. Shevrin and A.J. Ovsyannikov, Semigroups and their Subsemigroup Lattices (Kluwer, Dordrecht, 1996).

[15] A.J. Ovsyannikov, Lattice isomorphisms of completely 0-simple inverse semigroups, Semigroup Forum 62 (2001), 115-145.

Address:

Department of Mathematics, Statistics and Computer Science

Marquette University

P.O. Box 1881

Milwaukee WI 53201-1881

U.S.A.

Email: peter.jones@mu.edu 